\title{
SISTEMA DE ANÁLISE DE ATIVOS ATRAVÉS DE REDES NEURAIS DE MÚLTIPLAS CAMADAS
}

\author{
ASSET ANALYSIS SYSTEM USING \\ MULTILAYER NEURAL NETWORKS
}

Recebido 19/10/2011

Aceito $13 / 05 / 2012$

André Pacheco Miranda ${ }^{1}$ Rodrigo Luiz Antoniazzi

Luis Felipe Dias Lopes ${ }^{3}$

Marco Antonio Barbosa 4 Vânia Medianeira Flores Costa ${ }^{5}$

\section{RESUMO}

Quando investidores decidem se "aventurar" pelo mercado de renda variável, como pelo mercado de ações, buscam um método de ter mais segurança na tomada de decisão. Na prática, não há como saber quais ativos tornar-se-ão um investimento lucrativo. No mercado acionário, a Análise Técnica procura auxiliar o investidor na tomada de decisão. Para isso, utiliza-se de ferramentas e de métodos estatísticos para tentar predizer os movimentos do mercado. Este artigo apresenta o desenvolvimento de um Trade System robótico, utilizando um método heurístico. O sistema conta com uma rede neural multilayer perceptron, treinada com o algoritmo de retro propagação de erro, aproximando-se da análise técnica sem o fator emoção. Ao avaliar os resultados da rede neural, pode ser visto que a mesma obteve um resultado de $42,6 \%$ maior do que o diagnóstico da análise técnica.

Palavras-chave: Redes Neurais; Análise Técnica; Trade System.

\footnotetext{
1 Mestrando no Programa de Pós-Graduação da Universidade Federal de Santa Maria - PPGA/UFSM, Santa Maria, Rio Grande do Sul, Brasil. E-mail: andmirandapippi@gmail.com

2 Professor da Universidade de Cruz Alta - UNICRUZ, Cruz Alta, Rio Grande do Sul, Brasil. E-mail: rodrigoantoniazzi@yahoo.com.br

3 Professor do Departamento de Ciências Administrativas da Universidade Federal de Santa Maria - UFSM, Santa Maria, Rio Grande do Sul, Brasil. E-mail: Iflopes67@yahoo.com.br

4 Professor da Universidade Tecnológica Federal do Paraná - UTFPR E-mail: marco.cb@gmail.com

5 Doutora em Administração, Professora do Departamento de Ciências Administrativas da Universidade Federal de Santa Maria - UFSM, Santa Maria, Rio Grande do Sul, Brasil. E-mail: vaniaflores2006@yahoo.com.br
} 


\section{ABSTRACT}

When investors decide to "adventure" through stock markets they search for a method to provide safety on making decision. In fact, there is no precise way to know which stocks will became a profitable investiment. Technical analysis is a discipline that support the investors on making decisions. Such a discipline uses a set of tools and statistical methods to forecast the market's movement. Such a paper presents the develpment of a robotical Trade System, using a heuristic method. The system has a Neural Network multilayer perceptron, trained with an algorithm for back propagation error. Thus, approaching to the technical analysis without emotional aspects, using the Neural Network forecast on supporting the decisions of a investor on stock market. In analyzing the results of the neural network can be seen that the neural network got a result of 42.6\% higher than the diagnostic of the technical analysis.

Keywords: Neural Networks; Technical Analysis; Trade System.

\section{INTRODUÇÃO}

Uma boa exploração do mercado acionário como um meio de minimização de risco, sempre foi ambicionada por vários investidores da mesma, para isso, buscam vários métodos matemáticos e computacionais com a finalidade de entenderem a imprevisibilidade dos títulos financeiros e de controlarem o risco na tomada de decisão (HISSA, 2008). Mas a escolha de um ativo seguro e lucrativo nunca é uma tarefa simples. Na maioria das vezes, a emoção é que toma a decisão pelo investidor.

Contudo, existem modelos matemáticos a fim de prever as oscilações do mercado financeiro com a maior realidade possível. O uso desses métodos e modelos como forma de antever as possíveis movimentações do mercado financeiro não é uma prática nova. Nas últimas décadas, essa foi uma área de grande interesse para a comunidade de Finanças e Inteligência Artificial (KIMOTO, 1990; STEINER, 1995; LAWRENCE, 1997).

Os sistemas para a previsão do mercado acionário vão desde as mais simples técnicas estatísticas, presentes nas análises técnicas (ROBERT, 2007), na lógica fuzzy, nos algoritmos genéticos (LAWRENCE, 1997), nos modelos de Markov (HASSAN, 2005), nos modelos econométricos (OLIVEIRA et. al., 2010; COELHO et. al., 2008), até nos métodos heurísticos mais complexos, como as Redes Neurais (LAWRENCE, 1997; KIM, 2004; HASSAN, 2005; PHUA 2000; SMITH \& GUPTA, 2000; OLIVEIRA, et. al, 2010). Nas áreas de Administração e de Economia, as redes neurais são geralmente utilizadas para previsão dos preços no mercado acionário em função do mapeamento não linear entre as entradas e as saídas da rede.

O comportamento não linear, aleatório e altamente complexo do mercado acionário configura-o, aparentemente, imprevisível (FARHI, 2006). Porém com os grandes avanços em relação a utilização de métodos de previsão em outras áreas e com base na grande imprevisibilidade do mercado financeiro, questiona-se: será que um método de previsão mais complexo, como as redes neurais, pode identificar oscilações no mercado financeiro, com base em variáveis de entrada extraídos da análise de mercado?

Por tanto, com o intuito de auxiliar o investidor na tomada de decisão, controlando o risco das operações e visando atender ao problema de pesquisa delineado neste estudo, objetivou-se desenvolver um trade system robótico, ou seja, um sistema que utiliza um método heurístico na tomada de decisão, a fim de antever os movimentos do mercado sem o fator emoção. Esse sistema utilizará variáveis extraídas da análise técnica e de cotações das ações, para sugerir ao usuário pontos de compra, venda ou sustentação de um ativo analisado, controlando o risco na tomada de decisão do investidor (pessoa física). Para validar o estudo, será comparado o poder de explicação 
do método heurístico com os resultados extraídos de uma análise técnica, com base, em um setup contendo o operador matemático Média Móvel Aritmética.

O sistema desenvolvido utiliza a rede neural perceptron de múltiplas camadas (MLP), treinada com o algoritmo de retro propagação de erro (backpropagation) com aprendizado supervisionado, aproximando-se da análise técnica, sem o fator emoção (VALENÇA, 2009), o que auxilia o investidor na tomada de decisão. Em relação à utilização de múltiplas camadas, denominadas camadas escondidas em redes perceptron, significa mais poder computacional, do que o uso de estruturas de redes neurais sem as mesmas (LUDWING JR. \& MONTGOMERY, 2007).

Este artigo está estruturado da seguinte forma: na seção 2, são descritas técnicas de análise financeira; na seção 3 , faz-se uma breve descrição de redes neurais; na seção 4 , é apresentado o sistema proposto; na seção 5, são apresentados alguns resultados; por fim, na seção 6 , são consideradas algumas conclusões.

\section{TÉCNICAS DE ANÁLISE FINANCEIRA}

Existem várias formas, técnicas para avaliar se o preço de uma ação condiz com o patrimônio da empresa, ou seja, se esta vai cair ou subir. Entretanto, duas delas destacam-se entre as demais: a análise fundamentalista e a técnica. Embora ambas tenham por objetivo identificar a direção dos preços de uma ação, diferem na sua forma de avaliação.

\subsection{Análise Fundamentalista}

A premissa básica da análise fundamentalista é de que o valor justo para uma empresa (e, por consequência, para suas ações) está relacionado à sua capacidade de gerar lucros futuros. Para avaliar a possível direção no preço da ação, a análise fundamentalista leva em consideração informações obtida junto às empresas, partindo do entendimento do contexto macroeconômico e do panorama setorial nos quais a companhia se insere (microeconômico).

A análise fundamentalista é um tipo de análise indicada para analistas altamente especializados (GRAHAM, 2004), que incidem em utilizar todos os agentes externos como: fatores econômicos; condições de oferta e procura; metodologias para definir o preço justo a ser pago; informações da empresa; notícias sobre as condições da empresa; balanços e visitas à empresa, diariamente (DEBASTIANI, 2008).

Com a utilização de todos os agentes externos de uma análise fundamentalista, podese ter uma ferramenta excelente para operar no mercado acionário. Mas pelo fato do analista utilizar um grande período do seu dia para o desenvolvimento das visitas diárias ou semanais às instituições financeiras, a análise fundamentalista configura-se impraticável, se, no dia a dia, o investidor exercer outras funções (NORONHA, 2009).

\subsection{Análise Técnica}

Já a análise técnica, ou análise gráfica, utiliza-se do comportamento das ações no passado, para avaliar as possibilidades de flutuações futuras. A análise técnica consiste em utilizar gráficos multiformes, conjugados a fórmulas matemáticas e estatísticas que envolvem uma série de figuras ou formações que localizam tendências. Para o desenvolvimento de uma análise técnica, utilizam-se ferramentas de localização e mapeamento dos gráficos com: as linhas de suporte e resistência, que marcam topos e fundos nos gráficos; as teorias gráficas dos candelabros japoneses; os indicadores de tendências, as médias móveis e os osciladores, entre outros';

'Para uma visão mais completa do tema sugere-se como leitura das referência Robert, (2007) e Noronha, (2009). 


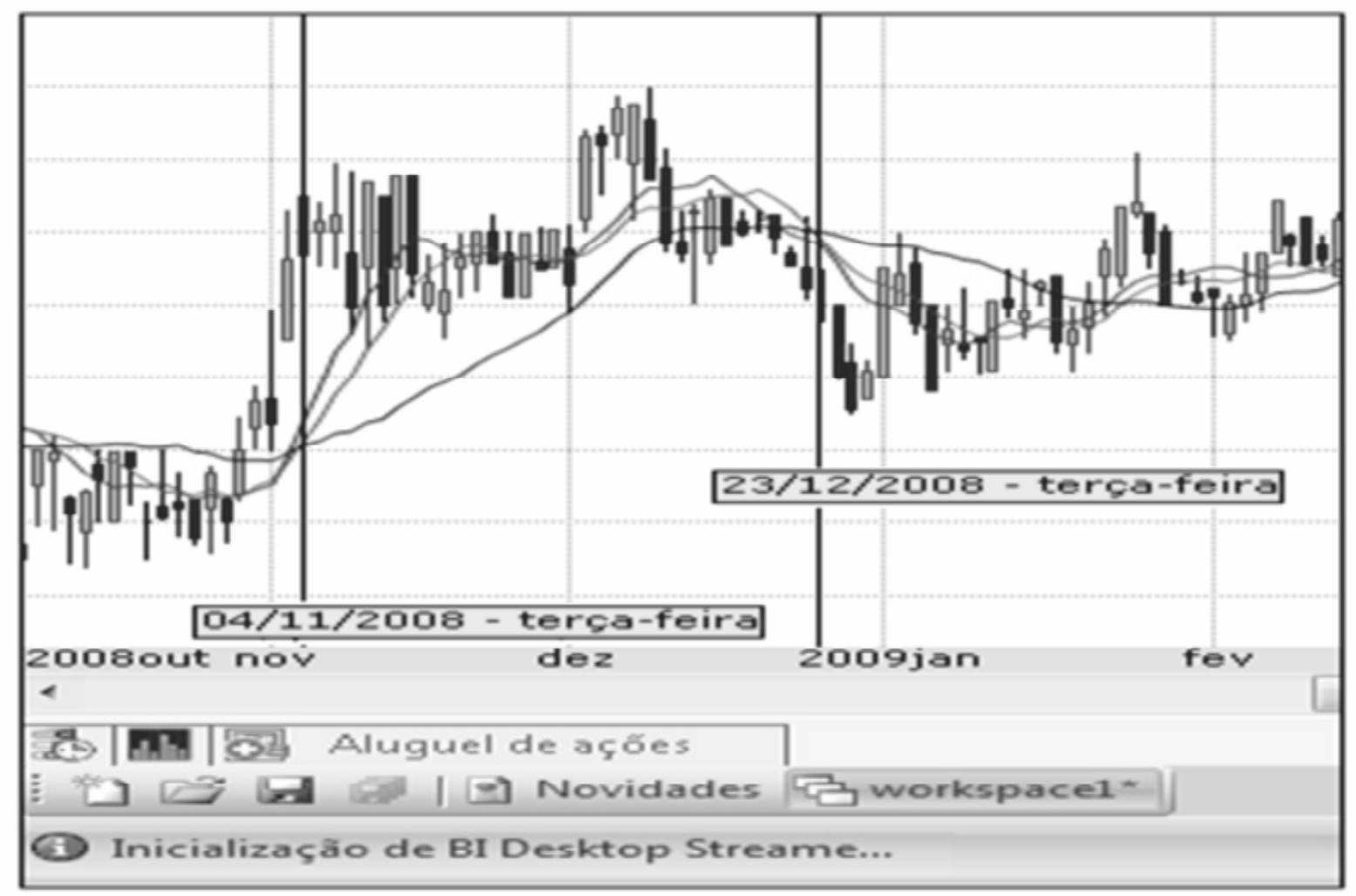

Figura 1: Analise Técnica do ativo NATU3.SA. Fonte: BANIFINVEST, (1999).

Na Figura 1, pode-se observar um gráfico contendo uma análise técnica do ativo preferencial da empresa de cosméticos Natura (NATU3.SA). Neste gráfico, foram utilizadas as técnicas descritas anteriormente para encontrar um ponto de compra e um de venda do ativo analisado, NATU3.SA. No dia 04/11/2008, quando a Média Móvel Aritmética (MMA), em cinza mais forte, sobrepôs as duas maiores, definiu-se como um ponto de compra. Na segunda linha vertical, destacada em cinza escuro, correspondente ao dia 23/12/2008, na qual a média mais alta, em cinza mais claro, sobrepôs às outras duas menores MMA, determinando-se como um ponto de venda e terminando-se um ciclo de compra e venda do ativo, o que conclui a análise técnica feita do dia 17/10/2008 até o dia 16/01/2009, para fins de comparação com os resultados da previsão da rede, neural na validação do trabalho.

\section{REDES NEURAIS ARTIFICIAIS}

O desenvolvimento da Rede Neural depende de diversos conceitos correspondentes a áreas distintas. A Psicologia contribuiu com os estudos das conexões entre neurônios biológicos e com o momento da aquisição do conhecimento no cérebro humano; a matemática forneceu a construção do neurônio matemático; e a Computação mesclou essas técnicas, automatizando-as, e criou a Neurocomputação, área que estuda as Redes Neurais Artificiais como um meio de transferir o aprendizado humano, para sistemas automatizados.

Então, uma rede neural artificial é composta por vários neurônios matemáticos que formam um modelo formal matemático para replicar o funcionamento e o processo de aprendizagem de uma cadeia de neurônios biológicos (JUDD, 1990). No neurônio biológico, há três componentes bastante significantes: os dendritos, os axônios e o corpo do neurônio (LUDWING JR. \& MONTGOMERY, 2007). Os dendritos têm a função de receber os estímulos e 
transmiti-los para as sinapses de outros neurônios. Os axônios são responsáveis pelos estímulos recebidos e transmitidos para as células. O corpo do neurônio é responsável pela coleta e combinações de informações vindas de outros neurônios (NASCIMENTO Jr. e YONEYANA, 2004).

O ciclo de funcionamento de um neurônio biológico, basicamente, é composto por três etapas: a primeira consiste em receber o estímulo das células, enviado pelas sinapses, e repassálo para o corpo do neurônio; a segunda consiste em compilar o estímulo e fazer os cálculos necessários; e a terceira etapa envolve repassar, pelos dendritos, o resultado calculado pelo corpo do neurônio para o próximo neurônio (LUDWING JR. \& MONTGOMERY, 2007).

Percebendo o funcionamento de um neurônio biológico, Warren McCulloch (1943), psicólogo, e Walter Pitts, matemático, desenvolveram o neurônio matemático. Eles combinaram os conceitos trazidos pela Psicologia, como o processo de aprendizagem de um neurônio biológico, e pela Matemática, como pesos e thresholds, para desenvolverem o neurônio matemático (MACCULLOCH e PITTS, 1943).

No neurônio matemático, as sinapses fazem a parte dos dendritos do neurônio biológico. O papel dos axônios é desempenhado, no modelo matemático, pelos bias (LUDWIG Jr. e MONTGOMERY, 2007). No neurônio biológico, o corpo do neurônio é responsável pelo processamento dos estímulos. Já no neurônio matemático esse papel é responsabilidade da função de transferência e de ativação (FARIA et al., 2008; LUDWIG Jr. e MONTGOMERY, 2007).

O processo de funcionamento de uma rede neural artificial, similarmente ao natural, é receber um ou mais estímulos de entrada e devolver um único sinal de saída para o próximo neurônios da camada posterior. A Figura 2, a seguir, exibe um Neurônio Matemático, corroborando os principais componentes de uma rede neural artificial.

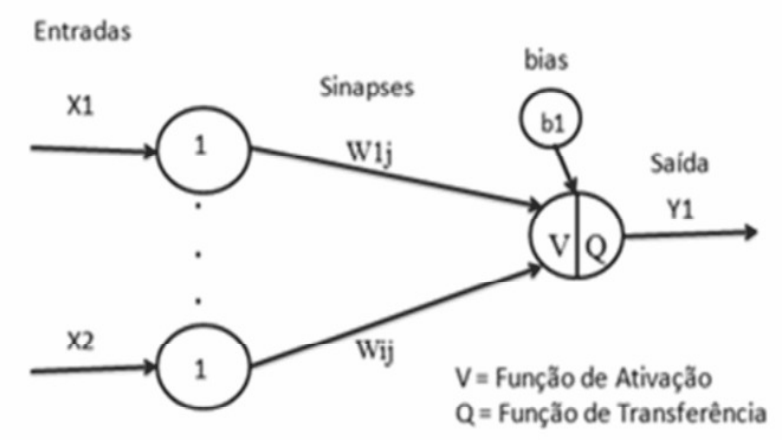

Figura 2: Neurônio Matemático. Fonte: Ludwig Jr. e Montgomery (2007); Nascimento Jr. e Yoneyana (2004); Ceretta et al. (2010).

Na Figura 2, são definidas as entradas em $\mathrm{Xi}$, as sinapses em $\mathrm{Wij}$, o bias em b1 e, em $Y$, a saída. O bias e as sinapses são modificados a cada interação, até chegar à saída desejada ou completar os ciclos escolhidos. As funções de ativação e transferência são responsáveis pela passagem do estímulo e pelo cálculo feito dentro do neurônio.

\subsubsection{Função de Ativação}

A função de ativação (2.1), proposta por Ludwig jr. e Montgomery (2007), é responsável por simular a primeira etapa dentro do neurônio, onde é feito o cálculo de ativação da estrutura, que está simulado pela letra $\mathrm{Vj}$, Equação 1, a seguir. Essa estrutura é desenvolvida dentro do neurônio matemático para corresponder ao primeiro cálculo dos estímulos que chegam pelos dendritos de um neurônio biológico. 
$v_{j}=\sum_{i=1}^{n} w_{i j} \cdot x_{i}$

Na Equação (2.1), o estímulo de todas as entradas recebidas pelo neurônio e os bias correspondentes são somados e multiplicados pelo resultado das camadas seguintes. Posteriormente, é passado o resultado para a segunda etapa dessa fase, a de transferência, que corresponde ao funcionamento do corpo do neurônio (LUDWIG Jr. e MONTGOMERY, 2007).

\subsubsection{Função de Transferência}

O corpo do neurônio biológico tem a função de calcular e repassar o estímulo aos próximos neurônios ou a função de saída final da rede. No neurônio matemático, a função de transferência é responsável pela função do corpo do neurônio biológico.

Portanto, a responsabilidade da transferência do estímulo recebido e emitido entre os neurônios matemáticos é auferida a três diferentes equações mais destacadas nas redes neurais, cada uma delas com suas características distintas. O Quadro 1, abaixo, demonstra as funções mais utilizadas (LUDWIG Jr. e MONTGOMERY, 2007).

\begin{tabular}{|ll|l|}
\hline $\begin{array}{c}\text { Gaussiana (1) } \\
\mathrm{Q}(\mathrm{v})=\mathrm{e}^{-\mathrm{v}^{2}}\end{array}$ & $\begin{array}{c}\text { Tangente Hiperbólica (2) } \\
\mathrm{Q}(\mathrm{v})=\tan \mathrm{h}(\mathrm{v})\end{array}$ \\
\hline & Sigmóide (3) \\
& $\mathrm{Q}(\mathrm{v})=\frac{1}{1+\mathrm{e}^{-\mathrm{v}}}$ \\
\hline
\end{tabular}

Quadro 1: Funções de Transferência. Fonte: Ludwig Jr. e Montgomery (2007)

A função de transferência utilizada na rede neural desenvolvida está simulada na equação 2.2, a seguir. Nesse caso, a Equação utilizada é a sigmóide. Nessa função, $Q(v)$ é o valor do resultado da função de transferência; vj(n) é o valor do resultado da função de ativação; e é a base natural de logaritmos. Em caso de aprendizado supervisionado, se a saída não for a almejada, compara-se com a saída desejada, obtendo-se o valor da equação de erro (VALENÇA, 2009).

$$
Q_{f}^{i}(v)=\frac{e^{-v_{j}(n)}}{\left(+e^{-v_{j}(n)}\right)},
$$

\subsection{Redes Neurais Perceptron}

Basicamente, todas as redes neurais são compostas por neurônio, camadas e sinapses, mas a grande diferença de uma rede neural para outra reside nas suas formas de treinamento / aprendizado e de correção das sinapses pelo erro. Então, o número de neurônios e camadas de uma RNA depende da sua funcionalidade ou do seu propósito.

Segundo Yonenaga e Figueiredo (1999), em 1958, Frank Rosenblatt construiu um dos primeiros tipos de redes neurais, a rede neural artificial perceptron. No seu trabalho, intitulado "The Perceptron: A Probabilistic Model for Information Storage and Organization in the Brain", Rosenblatt desenvolveu uma simulação computacional, corroborando que o reconhecimento de padrões é uma das características da retina. 
Essa rede desenvolvida por Frank Rosenblatt atualiza as sinapses com um método de ajuste supervisionado, quando o supervisor ajusta os parâmetros modificáveis da Rede Neural com base no valor do erro (YONENAGA e FIGUEIREDO, 1999). A menor representação da classe Perceptron como ajuste supervisionado é um neurônio na camada de entrada ligado a um neurônio na camada de saída.

Entretanto, logo após a publicação do trabalho de Frank Rosenblatt, em 1958, Marvin Minsky e Seymor Papert descobriram e provaram, no trabalho intitulado "Perceptrons", que as Redes Neurais de uma única camada não são tão eficientes assim (LUDWIG Jr. e MONTGOMERY, 2007). Eles confirmaram que, em algumas situações em que ocorre o problema da resolução da operação do OU exclusivo (XOR), a Perceptron não consegue resolver (VALENÇA, 2009). Com a publicação do trabalho de Minsky e Papert, desestimularam-se muitos pesquisadores da época na área de Redes Neurais (NASCIMENTO Jr. e YONEYANA. 2004).

\subsection{Perceptron Múltiplas Camadas (MLP)}

Mais tarde, com os estudos de John Holpfield, em 1982, que estabeleceu uma descrição da rede neural de uma lesma, os pesquisadores voltaram a se interessar pelas pesquisas nas áreas que compreendem as redes neurais (LUDWIG Jr. e MONTGOMERY, 2007; NASCIMENTO Jr. e YONEYANA. 2004). Então, Holpfield, em sua descoberta, propôs uma rede neural mais robusta, com algumas camadas entre a de saída e a de entrada, denominadas camadas ocultas. Com a criação dessa técnica, eliminou-se completamente o antigo problema do OU exclusivo (XOR).

Essa técnica, denominada Multilayer Perceptron, constitui-se de uma camada de entrada com tantos neurônios quanto forem os pesos de entrada, de uma ou de várias camadas ocultas e de uma camada de saída, com o número de neurônios igual ao número de pesos de saída (VALENÇA, 2009; GONÇALVES, 2005; NASCIMENTO Jr. e YONEYANA. 2004).

As Redes Neurais Artificiais de Múltiplas Camadas são extremamente precisas em suas respostas. A grande vantagem de utilizá-las é o conceito de que uma Rede Neural imita o cérebro humano, isto é, transcreve os atos de aprendizagem com erros e acertos em suas tentativas (ANITHA et al., 2012). Porém as Redes Neurais Perceptron, na prática, não conseguem fornecer soluções para problemas muito complexos sozinhas. Então, o algoritmo de retropropagação do erro com aprendizagem supervisionada tem o papel de ajudá-las nessa tarefa (LUDWIG Jr. e MONTGOMERY, 2007; SCHALKOFF, 2001).

\subsubsection{Retropropagação do Erro com Aprendizagem Supervisionada}

Há vários algoritmos que produzem o aprendizado para as redes neurais artificiais (LUDWIG Jr. e MONTGOMERY, 2007). O algoritmo de retropropagação do erro com aprendizagem supervisionada é um deles. Este é utilizado para controlar o erro e a taxa de aprendizado em redes neurais que necessitam de um supervisor como as perceptros.

Esse treinamento supervisionado, em relação à sua aplicação e ao seu desenvolvimento, pode ser classificado de duas formas totalmente distintas: o método de treinamento com supervisor forte e o método de treinamento com supervisor fraco. Então, quando a supervisão se caracterizar como forte, fornece a saída desejada para a rede neural conforme for o seu propósito, logo, aprendizagem supervisionada. Quando o supervisor não ajusta o erro do algoritmo pela saída desejada, o mesmo é chamado de supervisão fraca, portanto, o algoritmo de aprendizagem é não supervisionado.

Como mencionado anteriormente, o algoritmo utilizado pelas redes neurais perceptron é o de retropropagação de erro com aprendizagem supervisionada pelo fato de o supervisor fornecer a saída desejada e controlar o erro da rede. No entanto, segundo Valença (2009), para desenvolver o funcionamento desse algoritmo, depende-se de quatro passos. 
O primeiro passo começa com o vetor de valores de entrada, que inicia na primeira camada de neurônios. No segundo passo, são realizados os cálculos de ativação e de transferência, repassando o resultado à entrada do neurônio, na camada seguinte, prosseguindo até chegar à última camada de neurônios. No terceiro passo, na última camada, é calculada a diferença entre a saída desejada e a saída calculada pela rede neural. Esse processo de comparação é chamado de aprendizagem supervisionada com o supervisor forte. 0 quarto passo será feito se o resultado do erro não for satisfatório, pois é nesse passo que ocorrerá a correção das sinapses e bias dos neurônios.

Essa correção, chamada de retropropagação do erro, ocorrerá se o cálculo do erro do neurônio de saída e a taxa de aprendizado não forem satisfatórios. Dessa forma, os valores das sinapses e bias de cada neurônio serão corrigidos de trás para frente, ou seja, do último neurônio da última camada para o primeiro neurônio da primeira camada.

O grande propósito de utilizar um algoritmo com retropropagações do erro com aprendizagem supervisionada consiste em ajustar a previsão, de acordo com o erro, entre a saída desejada e a saída calculada pela rede neural. Se o erro entre os dois parâmetros não for aceitável, colocam-se novos pesos para as sinapses e bias do neurônio. Mas esse erro não pode ser inferior ao escolhido, ou ocasionará uma parada forçada da rede neural, e novos valores terão que ser escolhidos (LUDWIG Jr. e MONTGOMERY, 2007; GUIMARÃES, 2008). A seguir, na Equação 2.3, pode-se verificar a equação do cálculo do erro utilizada.

$$
e_{j}(n)=d_{j}(n)-y_{j}(n) \text {, }
$$

Na qual: dj(n) é o valor do erro desejado; yj(n) é o valor do erro calculado pela Rede Neural; $n$ é um valor real. Em uma rede neural de múltiplas camadas, utiliza-se o valor de erro para definir uma parada aceitável. A rede neural artificial procurará aproximar-se desse valor ou, até mesmo, igualá-lo em alguns casos. Portanto, para medir o desempenho do treinamento, diminuise o valor desejado do valor calculado do neurônio. A diferença indica o quanto a rede aprendeu com o treinamento supervisionado e, somente se seu erro ou seu desempenho forem aceitáveis, será considerada treinada (VALENÇA, 2009; LUDWIG Jr. e MONTGOMERY, 2007).

\subsubsection{Treinamento, Validação e Verificação}

Segundo Ludwig Jr. e Montgomery (2007), para que uma rede neural tenha credibilidade em seus resultados, a mesma necessita de três passos sequenciais da implementação: o primeiro passo é o desenvolvimento correto das redes utilizadas; o segundo passo é a ordem dos cálculos dentro do neurônio; e o terceiro é do ajuste dos bias e sinapses do neurônio no treinamento.

Após o desenvolvimento de uma rede neural, há três etapas chamadas de treinamento, que fornecem calibração dos parâmetros e definição do ponto ótimo de parada da rede: o treinamento / aprendizado, a validação cruzada e a verificação (VALENÇA e LUDERMIR, 2007; SAHOO et al., 2012).

O treinamento / aprendizado da rede neural Perceptron de múltiplas camadas (MLP) leva em consideração uma regra formal, a utilização de informações do supervisor da rede. Este atualiza os parâmetros modificáveis da rede neural, como os bias, a taxa de aprendizado, o número de ciclos, o valor do erro e as sinapses, para chegar a um resultado aceitável de erro. A participação do supervisor pode ser classificada em métodos de treinamento forte e fraco.

Como se expôs anteriormente, quando a participação for forte, o supervisor fornece um conjunto de valores de entradas correlacionados com um conjunto de valores de saída desejada na camada de saída. Esse método corresponde ao algoritmo de Back-Propagation, no qual o erro é retropropagado da saída para a entrada descrita anteriormente, fazendo com que a rede "imite" o supervisor (NASCIMENTO Jr. e YONEYANA. 2004; SCHALKOFF, 2001). 
A validação cruzada é um ponto muito importante para o treinamento das redes neurais. Com a validação, pode-se saber exatamente quando a rede neural está precisamente treinada para uma nova série de dados. Nesse estágio, tem-se uma certeza de parada, ou seja, descobre-se se a rede neural não está supertreinada para a entrada correspondente (VALENÇA e LUDERMIR, 2007), pois essa rede deve ser tão generalizável quanto possível.

$\mathrm{Na}$ validação cruzada, utilizam-se três equações para cumprir os objetivos citados anteriormente: o Erro Médio Quadrado, apresentado na Equação 2.5; o Erro Médio Global, apresentado na Equação 2.6; e o Erro Padrão de Predição, apresentado na Equação 2.7, a seguir. Com essas equações, procura-se um resultado aceitável de 0,08 a 0,05 por ciclo no Erro Médio Global, um resultado de 0,008 a 0,005 por ciclo no Erro Médio Quadrático e um valor de 0,008 a 0,005 por ciclo no Erro Padrão de Predição (VALENÇA, 2009; GUIMARÃES, 2008).

$$
\begin{aligned}
& E M G=\frac{1}{N} \sum_{p=1}^{N} Z_{p}-Z_{0} ; \\
& E M G=\frac{1}{N} \sum_{p=1}^{N}\left(Z_{p}-Z_{0}\right) ; \\
& E M G=\sqrt{\frac{1}{N} \sum_{p=1}^{N}\left(Z_{p}-Z_{0}\right) .}
\end{aligned}
$$

Nas quais: Zp é o valor previsto; Z0 valor corrigido; $N$ é o número de valores do conjunto de verificação, tal que $\mathrm{N}$ é um valor pertencente a todos os números naturais $\mathrm{e}$ positivos IR+;

O último quesito a ser avaliado no treinamento é a Verificação, momento em que há uma comparação entre o Erro Médio Quadrado da Verificação e o Erro Médio Quadrado da Validação Cruzada. Nesse estágio, é possível avaliar o desempenho do modelo utilizado e obter o ponto exato em que a rede neural deixa de estar treinada.

A partir do ponto em que as entradas da rede neural na Validação Cruzada resultam um Erro Médio Quadrado afastado do Erro Médio Quadrado da Verificação, são descartados os valores seguintes de entrada da Rede Neural. Então, a rede está treinada até aquele exato momento, pois ainda consegue reconhecer as características ou os padrões pelos quais foi treinada (VALENÇA, 2009).

\subsubsection{Topologias da Rede Neural}

A Topologia de uma rede neural é a soma do número de neurônio nas camadas de entrada, nas camadas escondidas (no caso das redes perceptrons) e nas camada de saída. A topologia que se enquadra melhor ao problema somente é verificada no treinamento, com o método de tentativa e com erro descrito em Sahoo et al., (2012).

A comparação com outras topologias testadas no treinamento consistem em expor qual se adere melhor ao plano de resultados almejados, quais as entradas, as saídas e o tipo de rede neural utilizado (VALENÇA, 2009; LUDWIG Jr. e MONTGOMERY, 2007; GONÇALVES, 2005). Por exemplo, redes com a topologia 2-3-3, como pode ser visto na Figura 3, a seguir. 


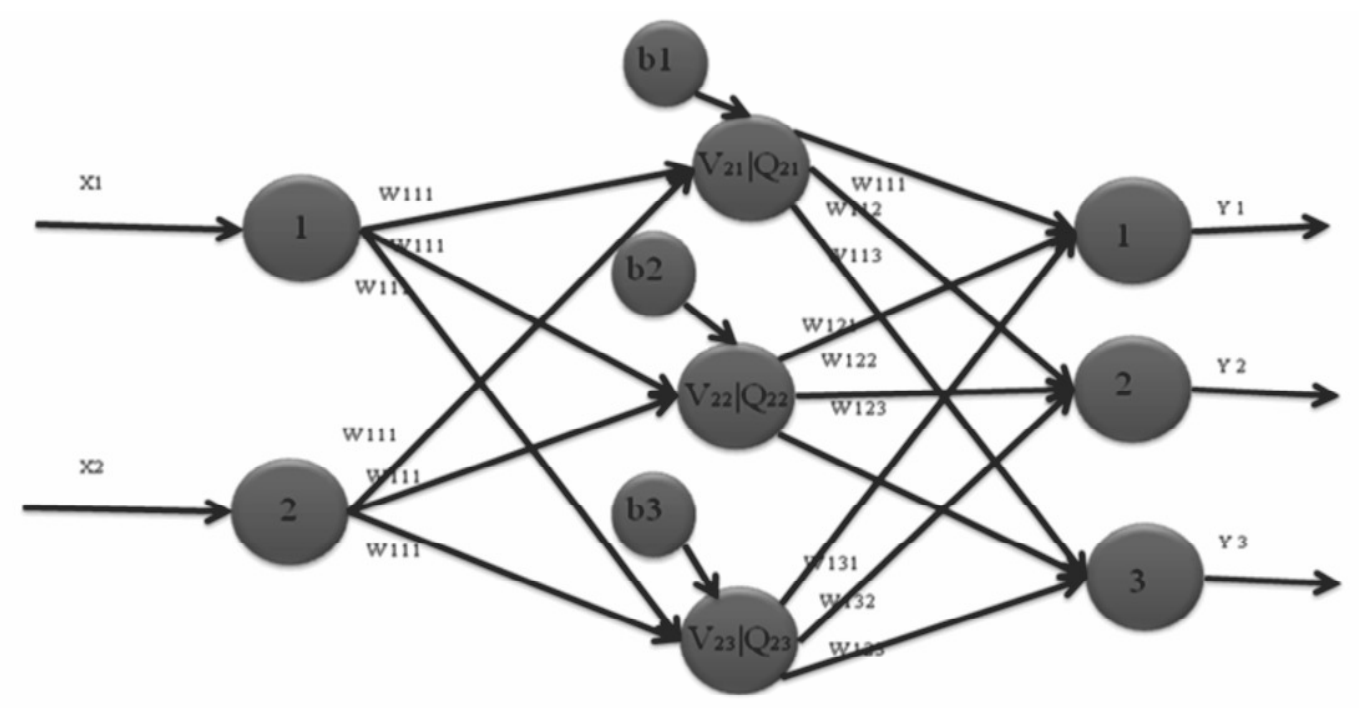

Figura 3: Topologia Rede Neural Artificial MLP. Fonte: Organizada pelo autor

\section{SISTEMA DE APOIO À DECISÃO}

Neste trabalho, foi construído um sistema de apoio à decisão voltado, principalmente, aos investidores com pouco ou nenhum conhecimento de técnicas de análise do mercado acionário. A rede neural presente no sistema foi desenvolvida na linguagem Java.

O sistema utiliza uma rede neural perceptron de múltiplas camadas para suas tomadas de decisões. Dessa forma, o sistema assegura maior garantia de sua saída. A rede aprende com o comportamento passado de um ativo específico e faz um prognóstico de comportamento futuro deste, fortalecendo a decisão do usuário e melhorando a confiabilidade da análise.

O objetivo da construção da rede neural, descrita neste trabalho, é construir um rastreador de tendências que apóie a tomada de decisões de um investidor na bolsa de valores, ou seja, auxilie-o a localizar o momento certo de compra e venda da ação analisada.

No treinamento da rede neural, foram testados trinta intervalos de saída desejada; cem taxas de aprendizado; dezenove modificações da topologia da rede neural, de dois neurônios até vinte e um neurônios na camada oculta; dez mil modificações do número de ciclos utilizado na rede neural, com o intuito de garantir que a solução encontrada seja tão generalizável quanto possível.

\subsection{Levantamento dos Dados}

Para as entradas da rede neural, serão utilizados 240 cotações do ativo NATU3.SA de 01/02/2008 à 19/01/2009 disponíveis no site da Bolsa de Mercadorias \& Futuros (BM\&F), onde foram divididas em quatro categorias, em proporções distintas para: a saída desejada; o treinamento / aprendizado; a validação cruzada e a verificação.

O treinamento / aprendizado satisfez 50\% das cotações obtidas de um total de 240 cotações. A validação cruzada correspondeu a $25 \%$ do total, e a verificação correspondeu aos $25 \%$ restantes. A saída desejada foi desenvolvida a partir de uma análise técnica. A escolha dos pontos de compra e venda correspondeu a uma análise do gráfico da Figura 1, em que um ponto de compra foi caracterizado de " 0 " no vetor de saída desejada da rede e de " 1 " para ponto de venda do ativo. Os demais pontos foram dispostos com coerência gradual para o mesmo intervalo, quando possível, acompanhando a posição do zero ou do um. 
O treinamento da rede neural foi realizado da forma descrita a seguir. Na camada de entrada, com três neurônios, foi recebido, no primeiro neurônio, um vetor de cotações diárias do ativo analisado (NATU3.SA) - Figura, 2. O segundo neurônio recebeu o tempo correspondente das cotações. E o terceiro neurônio recebeu um vetor de números para a calibração da função de transferência e, por consequência, um erro mais baixo, como mostra a Figura 4, a seguir, contendo as entradas e a topologia da rede neural utilizada.

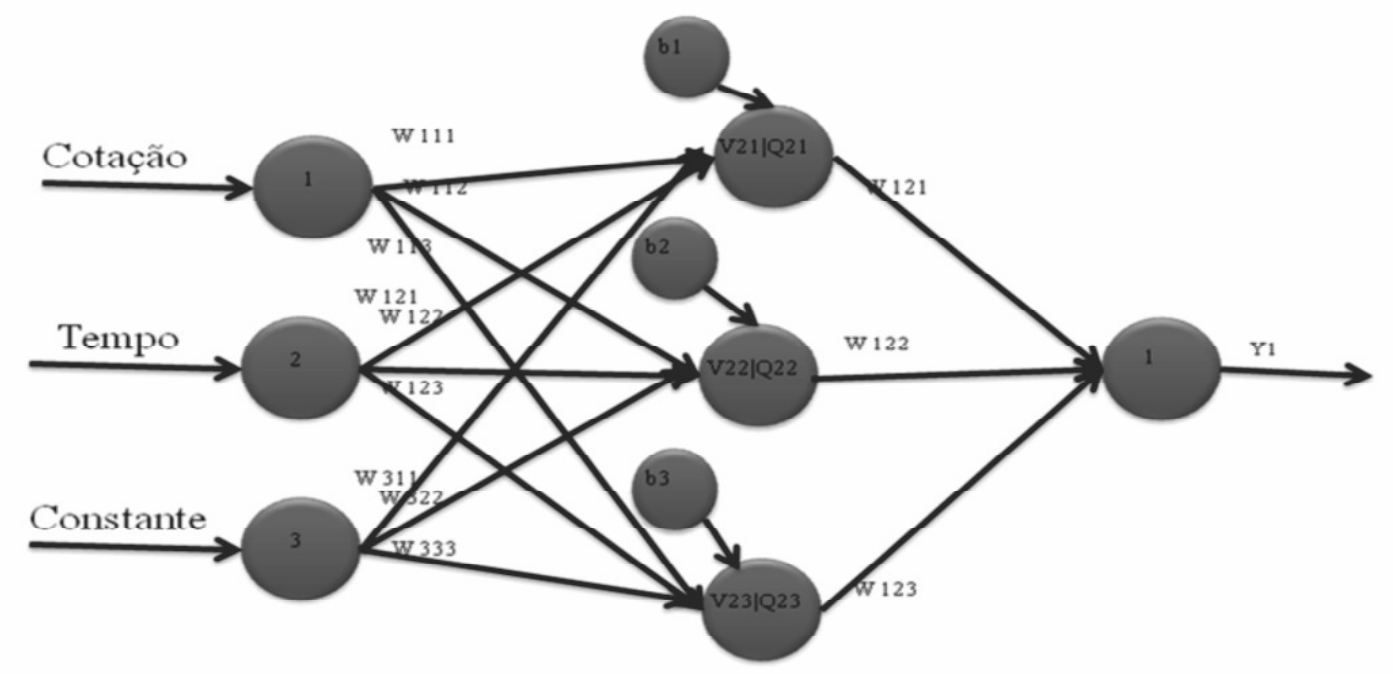

Figura 4: Entradas da Rede Neural Perceptron de Múltiplas Camadas. Fonte: Organizada pelo autor

\section{ANÁLISE DOS RESULTADOS}

Para o treinamento de uma rede neural, é necessário analisar resultados obtidos na modificação das entradas, valores, saída desejada, taxa de aprendizado, erro desejado, ciclo e topologia e métodos utilizados no treinamento e na validação da rede neural. Esse treinamento só estará completo se forem obtidos erros aceitáveis, descritos na Subseção 3.3.2. para todos os parâmetros.

Como forma de obter um erro pequeno, procurou-se obter uma baixa variação entre a saída desejada e a saída calculada pela rede neural, buscando sempre um menor Erro Médio Quadrático, Erro Médio Global, Erro Padrão de Predição. A rede neural desenvolvida neste trabalho constituiu-se de um aprendizado supervisionado.

$\mathrm{O}$ aprendizado supervisionado ocorreu com uma pequena margem de erro, pois o seu vetor de saída desejada constituiu-se de intervalos muito pequenos entre o primeiro valor de saída e os seguintes, abstendo de grandes variações entre um ponto e outro. Mas foram testados vários intervalos da variação dos valores de entrada na saída desejada da rede neural. Corroborandose com as afirmações anteriores, a que obteve o menor erro foi o menor intervalo testado o de 0,0295. A Tabela 1, a seguir, mostra essa correlação entre uma entrada do vetor de saída com a seguinte.

Portanto, pode-se perceber que com a utilização de intervalos de valores cada vez menores na saída desejada da rede neural perceptron de múltiplas camadas, proporcionaramse erros cada vez menores a cada interação. Para critérios de parada, foi utilizado o melhor erro obtido abaixo do menor erro aceitável, descrito na Subseção 3.3.2 do erro médio global. 
Tabela 1: Intervalo entre a Saída Desejada

\begin{tabular}{ccccc}
\hline & Intervalo de & Intervalo de & Intervalo de & Intervalo de \\
& 0,0295 & 0,050 & 0,070 & 0,090 \\
\hline $\begin{array}{c}\text { Erro Médio } \\
\text { Quadrático no Ciclo } \\
10000\end{array}$ & 0,002876 & 0,005299 & 0,023903 & 0,048138 \\
\hline
\end{tabular}

Fonte: Elaborada pelos autores.

A taxa de aprendizagem por interação foi escolhida a partir dos testes feitos na etapa do treinamento da rede neural. Nesse processo, ocorreu uma comparação entre os valores escolhidos e os resultados dos erros obtidos. Os critérios utilizados foram o menor erro por interação, o menor erro global, o menor erro médio quadrático e o menor tempo de execução.

Com os testes realizados, concluiu-se que a taxa de aprendizado por interação 20 , teve o melhor custo benefício entre as testadas, com o menor erro médio global no ciclo 10000, o menor erro por interação na posição 80 do vetor de saída do erro e o segundo menor tempo de execução total. A posição 80 do vetor de saída do erro foi escolhida aleatoriamente. Os resultados foram predispostos na Figura 5, na qual é mostrada a taxa que ocorreu menor erro médio global, menor erro médio quadrático e menor erro na interação escolhida.

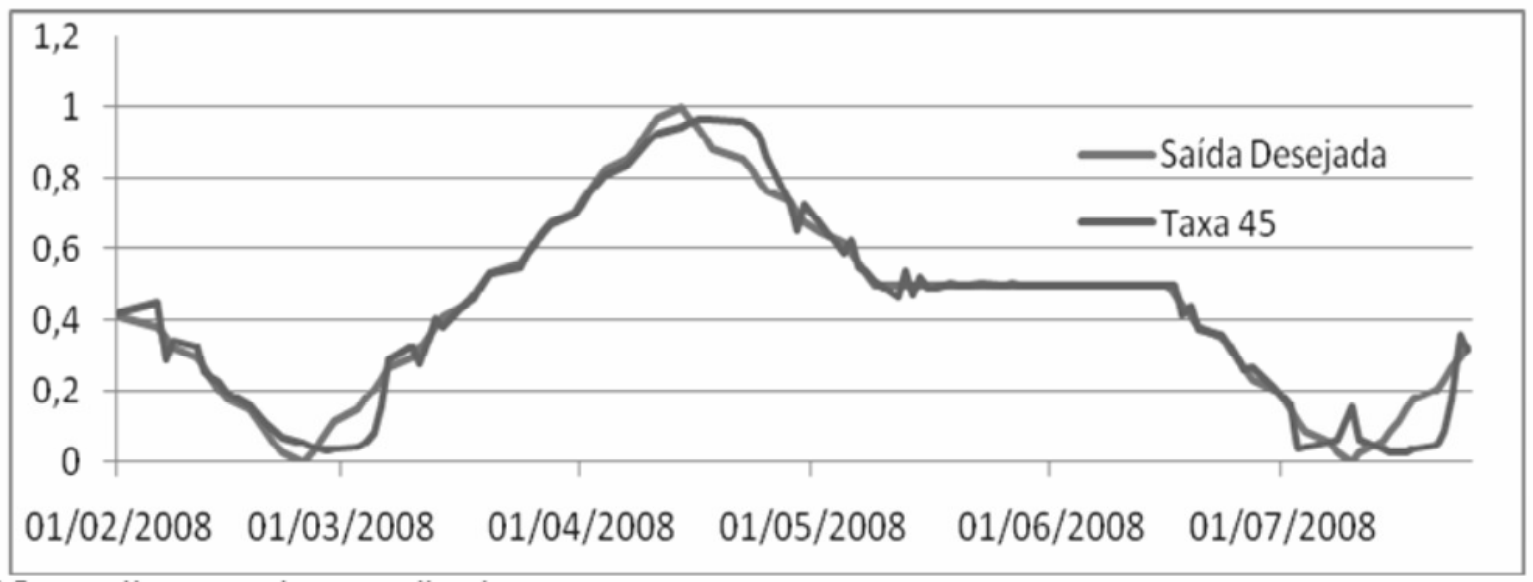

Figura 5: Gráfico melhor taxa de aprendizado. Fonte: Organizada pelo autor.

A rede neural conta com uma topologia 3-8-1, pois foi a melhor resposta no treinamento, dentre as 13 diferentes topologias testadas, variando entre 2 e 14 neurônios na camada oculta. A topologia que obteve um menor erro entre as testadas correspondeu a três neurônios na camada de entrada, a oito neurônios na camada oculta e a um neurônio na camada de saída. Nessa etapa, também foi observado quantos ciclos foram necessários para que a predição ocorresse em um erro médio quadrático aceitável, como mostra a Tabela 2, a seguir, com os resultados mais relevantes do Erro Médio Quadrático na Saída da rede neural. 
Tabela 2: Tabela da Topologia da MLP

\begin{tabular}{ccccc}
\hline & $3-4-1$ & $3-6-1$ & $3-8-1$ & $3-10-1$ \\
\hline Ciclo 100 & 0,002776 & 0,002325 & 0,001916 & 0,002007 \\
Ciclo 500 & 0,002574 & $\mathbf{0 , 0 0 1 9 1 1}$ & 0,001917 & 0,002733 \\
Ciclo 1000 & 0,002400 & 0,002259 & 0,001964 & 0,002722 \\
Ciclo 8000 & 0,002496 & 0,002533 & 0,002513 & 0,002714 \\
Ciclo 10000 & 0,002485 & 0,002518 & 0,002524 & 0,002713 \\
\hline
\end{tabular}

Fonte: Organizada pelo autor.

Na Tabela 2, pode-se observar que, na modificação dos parâmetros modificáveis, topologia e ciclo, os resultados do erro médio quadrático no ciclo 500, com a topologia 3-6-1, obtiveram o menor erro aceitável entre as demais testadas. A Figura 6, a seguir, demonstra a comparação do melhor resultado e do pior resultado alcançado, comprovando um dos objetivos do trabalho, que é mapear as oscilações do BOVESPA contraposta à saída desejada.

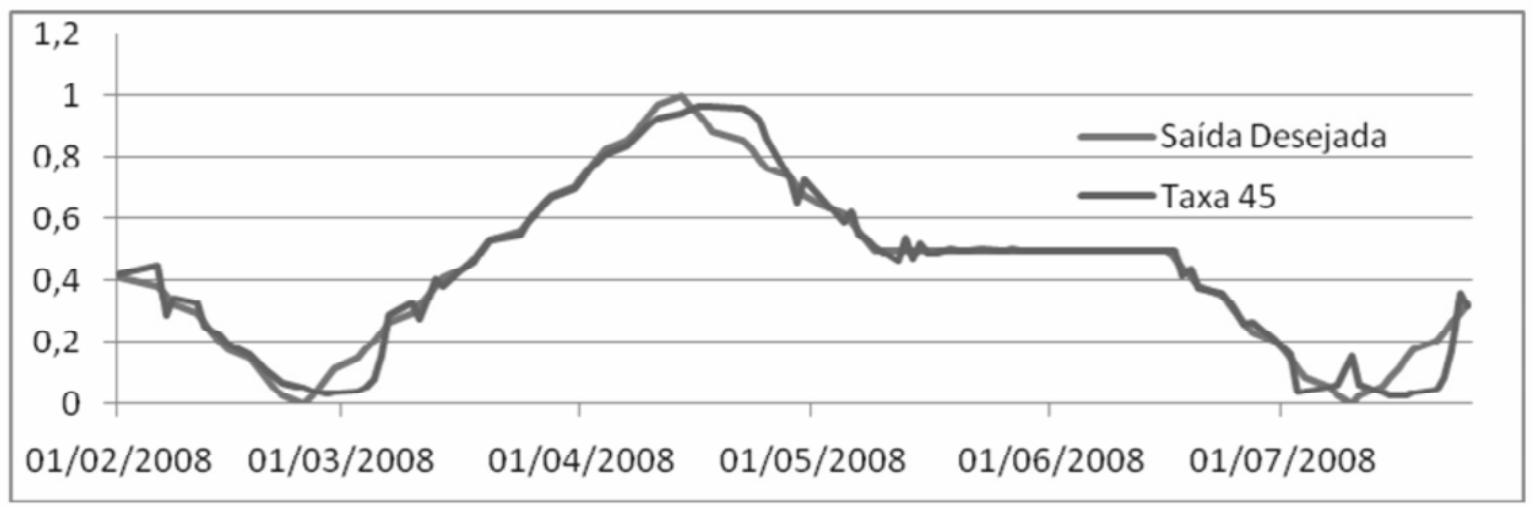

Figura 5: Gráfico melhor taxa de aprendizado. Fonte: Organizada pelo autor.

A rede neural conta com uma topologia 3-8-1, pois foi a melhor resposta no treinamento, dentre as 13 diferentes topologias testadas, variando entre 2 e 14 neurônios na camada oculta. A topologia que obteve um menor erro entre as testadas correspondeu a três neurônios na camada de entrada, a oito neurônios na camada oculta e a um neurônio na camada de saída. Nessa etapa, também foi observado quantos ciclos foram necessários para que a predição ocorresse em um erro médio quadrático aceitável, como mostra a Tabela 2, a seguir, com os resultados mais relevantes do Erro Médio Quadrático na Saída da rede neural.

Tabela 2: Tabela da Topologia da MLP

\begin{tabular}{ccccc} 
& $3-4-1$ & $3-6-1$ & $3-8-1$ & $3-10-1$ \\
\hline Ciclo 100 & 0,002776 & 0,002325 & 0,001916 & 0,002007 \\
Ciclo 500 & 0,002574 & $\mathbf{0 , 0 0 1 9 1 1}$ & 0,001917 & 0,002733 \\
Ciclo 1000 & 0,002400 & 0,002259 & 0,001964 & 0,002722 \\
Ciclo 8000 & 0,002496 & 0,002533 & 0,002513 & 0,002714 \\
Ciclo 10000 & 0,002485 & 0,002518 & 0,002524 & 0,002713 \\
\hline
\end{tabular}

Fonte: Organizada pelo autor. 
Na Tabela 2, pode-se observar que, na modificação dos parâmetros modificáveis, topologia e ciclo, os resultados do erro médio quadrático no ciclo 500, com a topologia 3-6-1, obtiveram o menor erro aceitável entre as demais testadas. A Figura 6, a seguir, demonstra a comparação do melhor resultado e do pior resultado alcançado, comprovando um dos objetivos do trabalho, que é mapear as oscilações do BOVESPA contraposta à saída desejada.

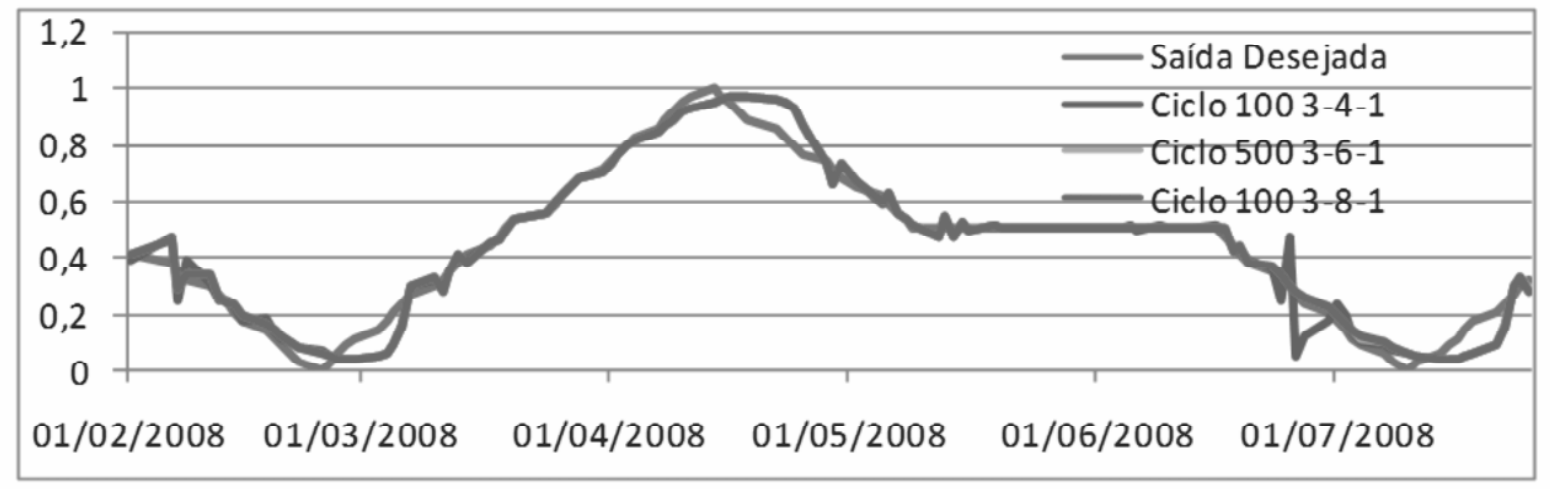

Figura 6: Gráfico Topologia e Ciclos Escolhido. Fonte: Organizada pelo autor.

O valor do erro desejado por interação foi escolhido a partir da aproximação do erro obtido com o erro desejado. 0 critério utilizado foi o de menor valor do erro calculado sem comprometer os resultados. A seguir, na Tabela 3, os resultados dessa comparação estão dispostos para intervalos do erro desejado aleatório.

\begin{tabular}{lccc}
\hline & $\begin{array}{c}\text { Erro Médio } \\
\text { Quadrático }\end{array}$ & $\begin{array}{c}\text { Erro Médio } \\
\text { Global }\end{array}$ & $\begin{array}{c}\text { Erro Padrão de } \\
\text { Predição }\end{array}$ \\
\hline Erro Desejado 0,70 & 0,003948 & 0,041783 & 0,062836 \\
Erro Desejado 0,50 & $\mathbf{0 , 0 0 1 9 1 1}$ & 0,035901 & 0,052274 \\
Erro Desejado 0,30 & 0,004779 & 0,053223 & 0,069130 \\
Erro Desejado 0,09 & 0,011786 & 0,078698 & 0,108565 \\
\hline
\end{tabular}

Fonte: Organizada pelo autor

Concluiu-se que o valor do erro desejado 0.50 obteve o melhor custo benefício entre os valores comparados, com a melhor aproximação do erro desejado e o menor erro propiciado pelo esforço da rede neural para alcançá-lo. Levando-se em consideração conjunto do treinamento e da validação, a rede neural com 500 ciclos foi considerada a mais satisfatória, pois foi a que obteve menos erros.

Para validar o sistema, foram comparadas duas formas de localização do Timing (melhor ponto de venda ou de compra de uma ação): a análise técnica e a análise do Trade System desenvolvido. Os métodos utilizados para a análise técnica foram os descritos na Seção 2. Já para a análise feita pelo Trade System foram utilizadas as técnicas no desenvolvimento de um método heurístico, assim como o treinamento e a validação descritos na Seção 3 e Sucessões.

Foram feitas duas análises do mesmo ativo, NATU3.SA, no mesmo período de tempo, de $17 / 10 / 2008$ ate 16/01/2009, com o intuito de observar qual análise tem a melhor disponibilidade de predição: a análise de um investidor humano, que utiliza seus anseios para sua tomada de decisão, ou a análise de um sistema robótico, que toma suas próprias decisões no mercado acionário. 
Como pode-se observar na Seção 2, foi feito uma análise técnica de um ponto de compra do ativo escolhido no dia 04/11/2008, por $\mathrm{R} \$ 19,50$ e de um de venda no dia 23/12/2008, por $\mathrm{R} \$ 21,22$, com base nas técnicas da Subseção 2 como mostra a Figura 1. Também foi feita uma análise do mesmo ativo no mesmo período de tempo, no dia 20/10/2008, pela rede neural descrita na Seção 3 e Sucessões, por $R \$ 17,02$ e uma análise de venda no dia 8/12/2008, por $R \$ 23,00$, na qual não foi possível observar indícios de uma previsão ascendente com base nos métodos estabelecidos na Seção 2, como mostra a Figura 6, a seguir.

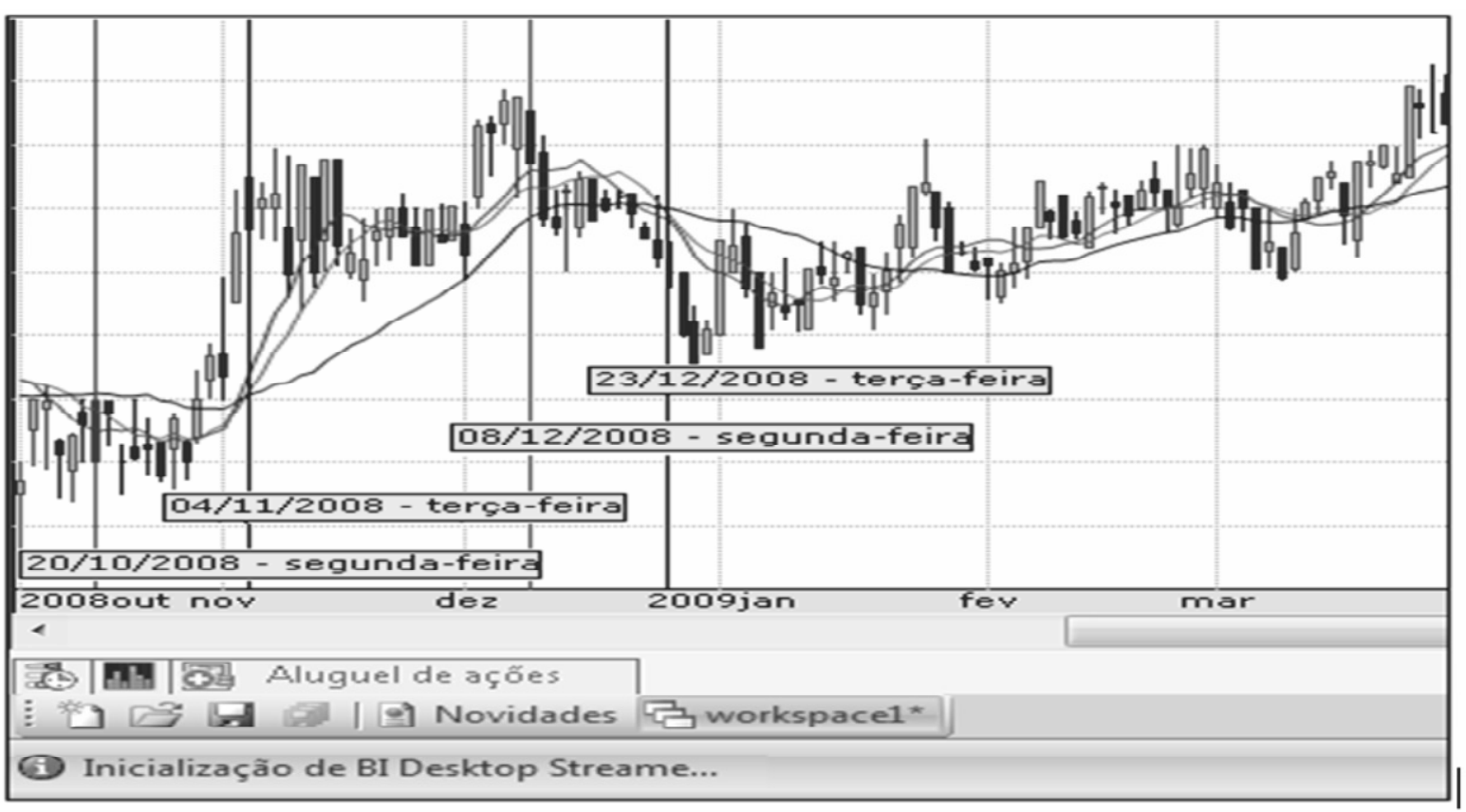

Figura 6: Comparação dos Pontos de Venda e de Compra. Fonte: BANIFINVEST, 1999.

Na Figura 6, após o ciclo de compra e venda concluído, é possível observar que a análise do Trade System obteve melhores resultados, pois, nela, é possível determinar pontos de compra e venda que não puderam ser identificados com somente a utilização da Análise Técnica, com base nos conceitos da Subseção 2 . Com os resultados da comparação entre a análise técnica e a análise do trade system, também se observou que o desempenho apresentado na análise técnica não obteve retornos negativos, mas os mesmos eram apenas menores.

\section{CONSIDERAÇÕES FINAIS}

Este trabalho obteve resultados positivos na predição de oscilações da ação analisada, com a utilização de uma rede neural artificial perceptron de múltiplas camadas, pois todos os parâmetros da fase do treinamento obtiveram erros aceitáveis, descritos na Subseção 3.3.2, para a equação do Erro Médio Global, do Erro Médio Quadrático e do Erro Padrão de Predição. Também se pôde examinar que o treinamento da rede neural foi aprimorando-se a cada novo parâmetro modificado pelo supervisor, obtendo um resultado altamente satisfatório de 0,001911 na validação da rede neural.

Então, com os resultados altamente satisfatórios obtidos da rede neural com ciclo 500, topologia 3-8-1, taxa de aprendizado 20, erro desejado 0,50 e intervalo de 0,0295, pôde ser construído um sistema de apoio à decisão para apoiar investidores na escolha de pontos de compra e venda de ativos no mercado acionário. Vários testes, com diferentes valores de variáveis, 
foram realizados para validar o sistema. O uso deste com o foco no ativo NATU3.SA fez com que as operações lucrativas sobrepusessem às operações com prejuízo no médio e longo prazo, em mais de $90 \%$.

$\mathrm{Na}$ analise dos resultados do Trade System, foi mostrado um ponto de compra do ativo NATU3.SA, no dia 20/10/2008, por $\mathrm{R} \$ 17,02$ e venda, no dia 8/12/2008, por $\mathrm{R} \$ 23,00$, tendo um retorno de $\mathrm{R} \$ 5,98$ por ação. Esse resultado, quando comparado ao desempenho do exame da análise técnica tradicional no mesmo período de tempo, indicou um resultado $42,6 \%$ superior a análise técnica tradicional.

$\mathrm{Na}$ analise da analise técnica tradicional, Subseção 2.2, observa-se claramente que os sinais de compra e venda tornam-se visíveis muito depois dos sinais dados pelo sistema apresentado neste artigo, tornado o diagnostico da análise técnica tradicional com menor retorno, descrita na Seção 5. Como trabalho futuro, espera-se desenvolver um sistema que possa trazer resultados nas operações de compra e venda não só de um ativo, mas também de grande parte dos ativos que compõem o índice da bolsa de valores de São Paulo (Ibovespa), comparado com a análise técnica convencional respectiva. Como limitações do trabalho, pode-se considerar o fator do tempo proporcionado para o desenvolvimento do artigo e o espaço limitado do mesmo. 


\section{REFERÊNCIAS BIBLIOGRÁFICAS}

ANITHA, J.; VIJILA, C. K. S.; SELVAKIMAR, A. I.; INDUMATHY. A.; HEMANTH, D. J.. Automated multi-level pathology identification techniques for abnormal retinal images using artificial neural networks. In: Journal BJO: Clinical Science, v. 96, n. 10, p. 220-223, 2012.

BANIFINVEST. BI Desktop Streamer 5. São Paulo. 1999. Sistema para Analise Técnica, Disponível em (https://www.banifinvest.com. $\mathrm{br} / \mathrm{tr} / \mathrm{bi} /$ produtos/bidsbeta/index.jsp). Acesso em: 10 Dez. 2010.

CERETTA, P. S; RIGHI, M. B; SCHLENDER, S. G. Previsão do preço da soja: uma comparação entre os modelos ARIMA e redes neurais artificiais. Revista Informações Econômicas, Instituto de Economia Agrícola. São Paulo. v.40, n.9. p 15-27. 2010.

COELHO, L. S.; SANTOS, A. A. P.; COSTA JR, N. C. A. Podemos prever a taxa de cambio brasileira? Evidencias empírica utilizando inteligência computacional e modelos econométricos. Revista Gestão de Produção. São Carlos, v. 15, n. 3, 2008.

DEBASTIANI, C. A. Análise Técnica de Ações: Identificando Oportunidades de Compra e Venda. São Paulo: Novatec, 2008.

FARHI, M. O impacto dos ciclos de liquidez no Brasil: Mercados financeiros, taxa de câmbio, preços e política monetária. Revista Política Econômica em Foco. Campinas - SP, n. 7. 2006.

GRAHAM, B.; DODD, D. Security Analysis. McGraw-Hill. 2004.

GONÇALVES, E. B.. Análise de Risco de Credito com o Uso de Modelo de Regressão Logística, RedesNeurais ArtificiaiseAlgoritmosGenéticos. Dissertação (Mestrado em Administração) Departamento de Administração da Faculdade de Economia, Administração e Contabilidade da Universidade de São Paulo, São Paulo, 2005.
GUIMARÃES, Alaine M.. Modulo de Validação Cruzada para Treinamento de Redes Neurais Artificiais com Algoritmos Backpropagation e Resilient Propagation. Revista Publicatio UEPG, Ponta Grossa, v. 14, n. 01, 2008.

HASSAN, R. M. D.; NATH B. Stock Market Forecasting Using Hidden Markov Model: A New Approach. In: Proceedings in the 5th International Conference on Intelligent Systems Design and Applications (ISDA'05). IEEE Computer Society, 2005.

JUDD, J. S, Neural Network design and Complexity of Learning. MIT Press, USA, 1990.

KIM, K.; LEE, W. B.. Stock market prediction using artificial neural networks with optimal feature transformation. Neural Computing \& Applications. Springer-Verlag, 13: 255-260, 2004.

KIMOTO, T, K; ASAKAWA, M; YODA, M. Takeoka. Stock market prediction system with modular neural networks. In: Proceedings of the International Joint Conference on Neural Networks, volume 1, pages 1-6, 1990.

LAWRENCE, R. Forecasting stock prices using neural networks. Department of Computer Science, University of Manitobo, December 1997.

LUDWIG J. R.; O.; MONTGOMERY, E. Redes Neurais: Fundamentos e Aplicações com Programa em C. 1. ed. Rio de Janeiro: Ciência Moderna, 2007.

NASCIMENTO J.R., L C.; YONEYANA, T.. Inteligência Artificial em Controle de Automação. São Paulo: FAPESP, 2004.

McCULLOCH, W.; PITTS, W. A logical calculus of the ideas immanent in neurons activity. Journal: Bulletin of Mathematical Biophisics, Springer New York, v. 5, n. 4, 1943. 
NORONHA, M.. Analise Técnica: Teorias Ferramentas Estratégias. 7. Ed. Rio de Janeiro, EDITEC, 2009.

OLIVEIRA, A. C. S.; SOUZA, A. A.; LACERDA, W. S.. Aplicação de Redes Neurais Artificiais na Previsão da Produção de Álcool. Revista Ciência e Agrotecnologia, Lavras-MG. V. 34, n. 2, p. 279-284, mar./abr. 2010.

PHUA, P.K.H.; MING, D.; LIN, W.. Neural Network With Genetic Algorithms For Stocks Prediction. Fifth Conference of the Association of AsianPacific Operations Research Societies, 5th 7th July, Singapore, 2000.

ROBERT E. D.; MAGEE Jr., W.H.C.. Technical Analysis of Stock Trends. American Management Association, 2007.

SAHOO, A. K.; ZUO, M. J.; TIWARI, M. K.. A data clustering algorithm for stratified data partitioning in artificial neural network. Journal Elsevier: Expert Systems with Applications, v. 39, n. 8, p. 7004-7014, 2012.

SCHALKOFF, R. J. Artificial neural networks. SAGE Publication - Progress in Physical Geography. New York: McGraw-Hill, 2001.

SMITH, K. A.; GUPTA, J. N. D. Neural networks in business: techniques and applications for the operations researcher. v. 27. p. 1023-1044. Oxford: Computers \& Operations Research. Elsevier, 2000.

STEINER, M.. WITTKEMPER, H. G.. Neural networks as an alternative stock market model. Journal Neural Networks in the Capital Markets, chapter 9, p 137-148. John Wiley and Sons, 1995.

VALENÇA, M.. Fundamento das Redes Neurais: Exemplos em Java. 2a ed. Olinda: Livro Rápido - Elógica, 2009.
VALENÇA, M. J. S.; LUDERMIR, T. B. Explicando a Relação entre as Variáveis de uma Rede Neural - Iluminando a "Caixa Preta". In: XVII Simpósio Brasileiro de Recursos Hídricos. São Paulo, 2007.

YONENAGA, H.W; FIGUEIREDO, S. R.. Previsão do Preço da Soja Utilizando redes Neurais. 11 f. Trabalho de Conclusão de Curso (Programa de Pós-Graduação em Engenharia de Produção) Universidade Federal de São Carlos - Rod, São Paulo, 1999. 